\title{
The linear motion as a scenario for addressing relations between a function and its derivative
}

\section{Dr. Eliud Quintero, Tecnologico de Monterrey (ITESM)}

PhD in Innovative Education by the Monterrey Institute of Technology and Higher Education (ITESM). Degree in Mathematics by UANL, in Monterrey, Mexico. Interested in research areas such as educational technology for the learning of mathematics, teaching - learning of calculus, visualization, analysis of multimodal production of signs.

\section{Dr. Patricia Salinas, Tecnologico de Monterrey (ITESM)}

Full time Professor at Mathematics Department in Campus Monterrey, Tecnológico de Monterrey. Educational researcher with interest in the integration of technology for the learning of Mathematics. With a Bachelors Degree in Mathematics and 2 Masters Degrees, in Education with Mathematics Specialization. PhD in Mathematics Education since 2011. Member of the National System of Researchers SNI 1, CONACYT, México. Co-author of several textbooks for the teaching and learning of Calculus. 


\title{
The linear motion as a scenario for addressing relations between a function and its derivative
}

\begin{abstract}
In this paper we present some results of a study conducted with 65 engineering students in a first Calculus course. SimCalc MathWorlds ${ }^{\circledR}$ software was included in the design of a didactic sequence to incorporate the scenario of the motion of an object over a horizontal straight line. The software document that was designed to work in classroom, plays an important role for analyzing the graphical behavior of position and velocity functions. By dragging the velocity graph, the software acts with the corresponding change in the position graph. The students used the software in their laptops by pairs during the two weeks didactic sequence. The aim of the sequence is to set up some relationships between the behavior of velocity graph and position graph. In a conventional curriculum those relations refer to the positive (negative) sign, and increasing (decreasing) behavior of derivative function, corresponding to the increasing (decreasing) and concave upward (downward) behavior of the function. Software brings the scenario for learning those facts analyzing the real context of linear motion. As part of the study, an assessment instrument was designed in order to appreciate the students' appropriation of those relations. The instrument' items are classified by corresponding to the linear motion context, or corresponding to different real contexts (no motion), or without including any real context. They also consider the posing information of the item and of the answer, being described by text or by a graphic. Application of the instrument lead us to reflect that, once the appropriation is achieved through the motion context, it could be easier for students to apply it without connection with a real context. It also reveals the difficulties for interpreting graphical information based on the derivative function. These findings are part of the overall results of a doctoral dissertation concerning with the use of digital technologies for the learning of Calculus.
\end{abstract}

Keywords: Calculus learning, digital technologies, linear motion, real context, mediation.

Background

Digital technologies are important tools in our daily activities, and it looks easy to use them in classroom to support learning. According to Hillman ${ }^{1}$, a lot of research has been conducted, suggesting that these technologies can help students make connections to mathematical concepts and enrich their mathematical thinking. Great effort has been addressed to identify and measure student performance in terms of achievement. In doing so, there is a noticeable common practice of using technology for a better learning of the same mathematical content that has been taught before, without technology. But, should we assume to teach the same mathematical content? Or, could it be possible to prove some changes with the intentional integration of digital technology?

Calculus courses have been redesigned in our educational institution in northern Mexico. In this approach for the learning of Calculus, the concepts of derivative and integral are included in the first course, instead of the classic Differential Calculus course. The Fundamental Theorem of Calculus has been adopted as an initial state, giving a strategy to accomplish the practice of predicting values of a magnitude that is changing. This meaning allows the student to make sense 
of the concepts of derivative and integral (antiderivative) when dealing with the study of motion over a straight line $2,3,4$.

We have been evaluating different software for learning goals, fitting this innovative approach for Calculus. The need of a dynamic interaction between the user (student) and the tool (software) makes SimCalc MathWorlds (C) (hereon SimCalc) software with potential to transform the way mathematics can be learned ${ }^{5,6}$.

The main idea that motivated the creation of SimCalc project was to foster and enrich the Mathematics of Algebra and Calculus, and simultaneously give students an early access to important concepts. According to Burke, Hegedus y Robidoux ${ }^{7}$, the SimCalc design team (including researchers, software developers and teachers) sought not only improve existing school curriculum, but also transform it with activities that would not be possible without the use of technology. In order to do so, a key element in SimCalc is its representational infrastructure that allows observing different representations (graphical, algebraic, tabular) dynamically linked.

This feature of linked representations inspired us to use SimCalc providing a global picture of the notions of derivative (seen as velocity) and antiderivative (seen as position) through the scenario of a motion simulation and its graphical representation. Figure 1 shows the SimCalc graphical interface designed for the work presented here. There, two characters move in opposite ways over a straight-line in a cartoon world. It also shows the two dynamically linked graphs of velocity and position corresponding with the two characters in scene.

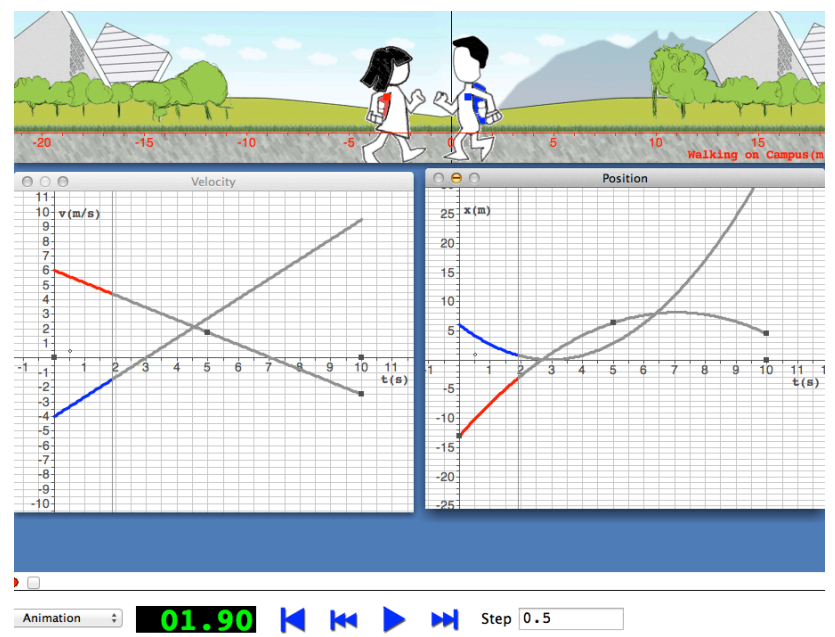

Fig. 1 SimCalc graphical interface for Uniformly Accelerated Motion.

The Fundamental Theorem of Calculus is a key element in the infrastructure of the software, in this way; velocity and position functions are linked dynamically according to this mathematical fact. Dragging velocity trigger changes in position and the dynamic visualization of those changes is a source for mathematical thoughts concerning graphs' behavior.

The scenario of Uniformly Accelerated Motion invites to think in features of the characters' animation, like moving to the right (left) progressively faster (slower), and try to figure out some possible relation between graphs and the simulation of motion. 
Past experience with this scenario has shown us that students do not visualize easily those relations on their own. We have been organizing a didactic experience including SimCalc to lead students into a visual process for discovering relations between graphs of velocity and position. The experience has resulted in a doctoral dissertation, and in this paper we focus in one of their findings.

\section{Rationale}

The theoretical background of the complete doctoral study is based on Vygotsky's sociocultural theory. Vygotsky ${ }^{8}$ explains how humans make use of environment tools to extend the operation of the memory beyond the biological dimensions. Humans incorporate external and artificial stimuli called signs. Relatively simple actions like tying a knot or mark signals on a stick, modify the internal structure of memory to be used to help remembering. With a superior psychological function Vygotsky refers a combination of a tool and a sign in the psychological activity of people. Internalization is the process for reconstructing internally an external operation.

Wertsch ${ }^{9}$ states two alternatives for the term internalization. As mastery, it refers to knowing how to use easily a mediation tool, and as appropriation it refers to taking something, which belongs to others, and make it self-owned. Rogoff ${ }^{10}$ highlights the importance of getting involved actively in social interaction in order to appropriating some aspects of the environment.

The notion of appropriation is relevant to describe the process by which students build mathematical meanings during the activity with SimCalc. Hegedus and Moreno - Armella ${ }^{11}$ propose the notion of co-action to emphasize the role of the environment in which the tool is being used, and attend the dialectical process between users, tool and environment. With this concept, we describe how the user guides actions over the environment, and in turn, is guided by the environment, as a fluid activity.

According to Moreno - Armella and Hegedus ${ }^{12}$ students do not passively wait to be defined by the environment of a learning situation, but through co-action, they can arrive to perform a wellstructured mathematical activity. So, although the environment can potentially lead to the emergence of meanings, it is not sufficient to fulfill this purpose. It is the action and observation on what varies or is invariant in the explorations, what makes effective the co-action between the learner and the environment.

In our study, the didactic sequence involving the use of SimCalc, provides the environment where the students, working in pairs, will be involved in a mathematical activity to propose and test their own conjectures, performing the right co-action with the software.

\section{Method}

The present work seeks to complement a broader study, as has also been addresses in Salinas and Quintero $^{13}$. We are concerned with the use of technology integrated to Mathematics learning. We look for a dynamic visual display that could support students' thoughts and reasoning dealing with graphs. Working on the $\mathrm{PhD}$ dissertation of one of the authors we took the challenge for the design of a didactic sequence trying to focus in the use of SimCalc as a mediator for the 
appropriation of the relations between velocity and position functions. A part of this study includes the application of an assessment instrument where several items consider transferring knowledge from linear motion to other real contexts. Findings with this instrument are the ones we share in here.

The research took place at a private university in northern Mexico. We decided to invite students from the three scholar groups of course Mathematics I for Engineering in charge of one of the authors. A total of 65 participants, about 19 years old, were up going a classroom with several technological resources facilitating the development of activities. In Figure 2 we observe an image of the classroom.

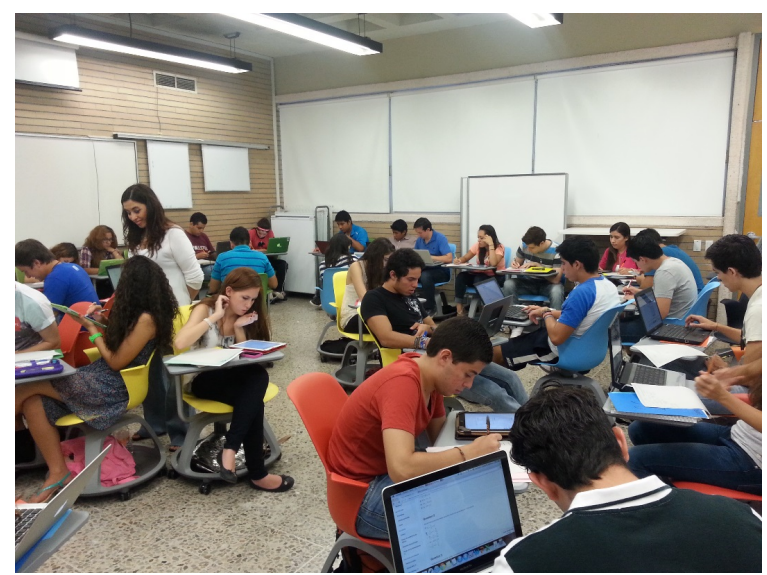

Fig. 2 Classroom equipped with technological resources.

The didactic sequence includes several activities performed in two weeks, we were working in four classroom sessions, an hour and a half each. We designed a set of activities within the SimCalc environment for the analysis of quadratic functions and their relation to linear functions, as proposed in Salinas et al. ${ }^{14}$.

It is included an initial session where teacher, in a lecture format, uses the software to make students aware of how the software allows an interaction with the graphs and how to interpret visual information from them. The latter was done with the particular scenario of uniform linear motion, where velocity graphs are horizontal, and it is just possible dragging graphs up or down, maintaining their horizontal property. The teacher also presents the document where students will do the activity directly in SimCalc. There, the velocity graph allows the change in slope by dragging the end point of the velocity segment. In Figure 3 we can observe a variety of situations that could emerge when dragging the velocity. 


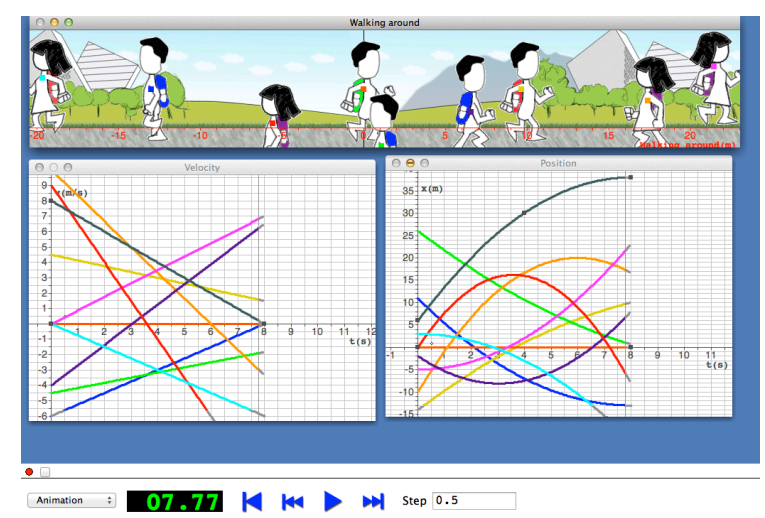

Fig. 3 A variety of situations in the SimCalc environment.

Our idea with the didactic sequence is to promote the organized interaction of the students with SimCalc in order reveal thoughts that could be identified as mathematical relations between the graphs. As discussed before in Salinas and Quintero ${ }^{13}$, we are looking to support a situated proof for those relations through SimCalc.

A mixed method allowed us to consider several information sources that could reveal the processes of appropriation by students when they interact with the software. It was important during the implementation phase, to take control of installation details of the software and also with our special request to students to generate video recordings of their laptops screens including video of themselves through the webcams while working and discussing the activity. This material has been widely analyzed, giving place to several qualitative information not discussed in this paper. Here we focus on the design and application of an assessment instrument that was applied in the final phase of the study to include some quantitative information.

The students were asked to join in pairs and work with one laptop on the SimCalc document along with a worksheet given to them, designed to help them to write down their results. In Figure 4 we observe the initial stage for the activity. In the SimCalc document they could drag velocity graph, observe the simulation of motion and analyze the changes in position graph, all this gives place to different features like the ones illustrated in last figure 3, but now the task is to fill the rows in the worksheet with any of the words provided in the six boxes below.

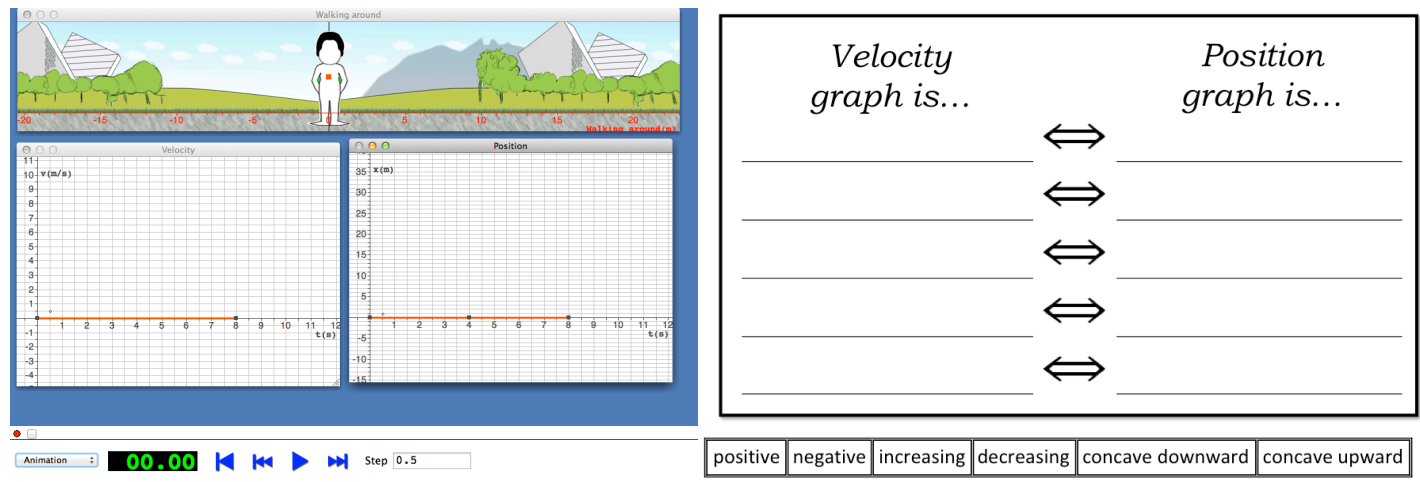

Fig. 4 SimCalc document and worksheet given to students. 
The words refer to features of the velocity graph that imply and are implied by the position graph. The table contains two columns including lines and arrows representing implications. Each row has a pair of lines with a double implication arrow between them. Each row represents a statement that the students may conjecture, supported on the co-action performed through SimCalc. During the activity we do not say if all the words should be used, or if they can be repeated, or how many rows should be filled.

The session class finalizes when students deliver their own video and their statements on the worksheet. The hour and a half has been enough time to perform what we described here. It is important to point out that not all the pairs of students arrive to the right relations between velocity and position graph. But it is considered in the didactic sequence the teacher's involvement in order to watch briefly the students' worksheet to inquire for the SimCalc image that made them fill certain wrong row. This invites students to work with SimCalc and discuss what could be wrong. Finally, before class finishes, every pair of students arrives to the right relations between velocity and position graphs, we summarize them in Table 1.

Table 1.

Qualitative relations between velocity graph and position graph.

\begin{tabular}{cc}
\hline Velocity (derivative) & Position (function) \\
\hline Positive & Increasing \\
\hline Negative & Decreasing \\
\hline Increasing & Concave upward \\
\hline Decreasing & Concave downward \\
\hline
\end{tabular}

The video recordings made by the students while they worked together have been analyzed in detail through transcripts; we used the analysis of speech and gestures in interaction. Several results arose and gave place to important qualitative information for the study, but in this paper we focus on the application of a quantitative instrument to assess the students' appropriation of the graphical relations between a function (position) and its derivative (velocity) mediated by the SimCalc environment. The instrument assess the recognition and application of the graphical relations in Table 1, when the context in which the situation is presented is not the linear motion, or even when no real meaning is associated with the function and derivative

\section{Assessment Instrument}

The assessment instrument was applied on paper in class, the week after the didactic sequence finished. It includes 14 items in a multiple answer format, and all dealing with information visualized through graphs or interpreted from a text description. Multiple answer formats allow more than one correct answer to an item. Although there could be more than one correct option for each item, all of them are independent of each other.

We assign a partial score (20\%) to each of the five options for a particular item. Score is assigned when a correct option is selected, and also when an incorrect option is not selected; whereas no score is assigned when incorrect options are selected, or also when correct options 
are not selected. In Table 2 we observe the scores assigned each time an option for a particular item is selected or not.

Table 2.

Score assigned to a type of selected option for a particular item.

\begin{tabular}{lcc}
\hline \multicolumn{3}{c}{ Score assigned to the choice of each option } \\
\hline \multicolumn{2}{c}{ Is selected? } \\
\hline Kind of option & Yes & No \\
\hline Correct & 0.20 & 0.00 \\
\hline Incorrect & 0.00 & 0.20 \\
\hline
\end{tabular}

The design includes seven types of items, named with the letters A to G. In Table 3 we explain the different types according to the posing information for the item (columns in Table 3 ) and the posing information for the options (rows in Table 3). We illustrate with some examples next.

Items Type A describe in text some information about certain magnitude that is changing, and the options are given with the graph of the rate of change of the magnitude. Items Type E pose the information showing the graph of a magnitude that is changing, and the options are given with the description in text interpreting the real context. Items Type D pose the information with the graph of the rate of change (derivative) of a magnitude, and the options give the information with the graph of the magnitude (function). The extra items Type G consider situations in which both graphs, function and derivative, are presented in the same coordinate system, and not apart, like SimCalc presents.

Table 3.

Structure of six types of items included on the instrument.

\begin{tabular}{ccccc}
\hline Item posing information: & $\begin{array}{c}\text { Context } \\
\text { description }\end{array}$ & $\begin{array}{c}\text { Derivative } \\
\text { (rate of change) }\end{array}$ & $\begin{array}{c}\text { Function } \\
\text { (magnitude) }\end{array}$ \\
\hline \multirow{2}{*}{$\begin{array}{c}\text { Options } \\
\text { posing } \\
\text { information: }\end{array}$} & $\begin{array}{c}\text { Context } \\
\text { description }\end{array}$ & - & Type C & Type E \\
\cline { 2 - 5 } & $\begin{array}{c}\text { Derivative } \\
\text { (rate of change) }\end{array}$ & Type A & - & Type F \\
\cline { 3 - 5 } & $\begin{array}{c}\text { Function } \\
\text { (magnitude) }\end{array}$ & Type B & Type D & - \\
\hline
\end{tabular}

For each of the seven Types of items, there is an extra feature that allows a new rating according to three types of contexts where the information has to be stated. It could be the real environment of Motion Context (MC) that has been studied in class through SimCalc, or it could be another real magnitude involved in Other Context (OC), or it could be mathematical information not related to real magnitudes, which we name as Without Context (WC).

In Table 4 we observe the 14 items (numbers from 1 to 14) classified according to the context (MC, OC, WC). The instrument considers three items without relation to a real context, seven items related to the motion context and four items where we introduced different real contexts than the linear motion. 
Table 4.

Distribution and quantity of 14 items that constitute the instrument.

\begin{tabular}{cccccccc}
\hline & A & B & C & D & E & F & G \\
\hline Without Context & - & - & - & 7 & - & 11 & 13 \\
\hline Motion Context & 1 & 3 & 5 & 8 & 9 & 12 & 14 \\
\hline Other Context & 2 & 4 & 6 & X & 10 & X & X
\end{tabular}

We illustrate as an example item 4. It is classified in Other Context (OC) because the posing information is a text description of a magnitude that is changing. It is also classified as Type B because the options given for answer are given through the graph of magnitude. Item is as follows: The level of water (in centimeters) of a tank is changing with respect to time (in minutes), so that at first decreases progressively slower until empty, and then grows increasingly faster until filled. Which of the following graphs of the level, does it adequately represents this situation? Options for this item are shown in Figure 5, in this case the correct options are b) and c).

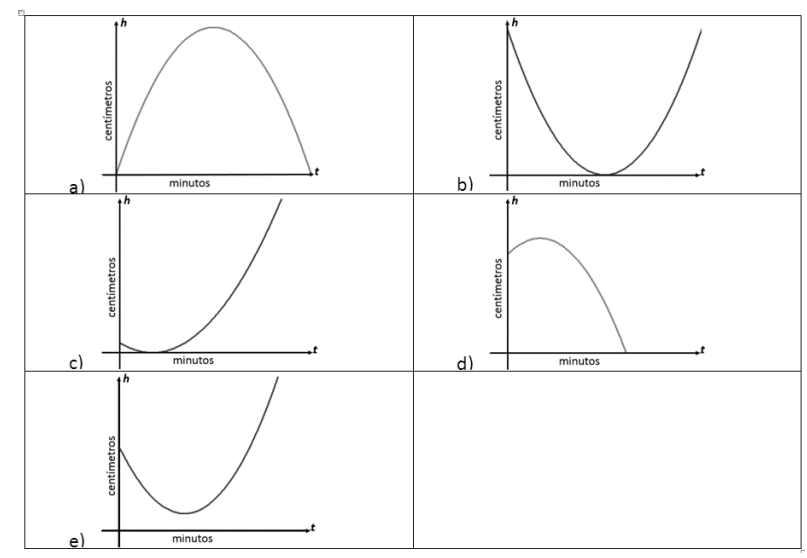

Fig. 5. Options for item 4 in the assessment instrument.

Additional contexts considered in the instrument are: the variation of the area of a rectangular field with fixed perimeter, the change in the water level in a tank, the temperature change of a material and the variation of the production cost of a certain article.

We apply the assessment instrument to the 65 study participants. Through the application, we determine the degree of reliability of the instrument using Cronbach's alpha coefficient ${ }^{15}$. This index is a measure of internal consistency. The score obtained is 0.7514 which means the reliability of the instrument is acceptable.

Results

Assessment instrument items were analyzed by a Mean Comparison Test using the software SPSS 20. Table 5 shows the average of the scores for each of the 14 items. The overall average achieved by the total study participants is 75.10 , with a standard deviation of 15.32 points. This alludes to moderately high scores with a wide distribution between them.

Table 5 .

Scores obtained for each item. 


\begin{tabular}{cccccccc}
\hline Type de context & MC & OC & MC & OC & MC & OC & WC \\
Item & 1 & 2 & 3 & 4 & 5 & 6 & 7 \\
\hline Average & 91.08 & 66.77 & 66.77 & 90.46 & 50.15 & 39.38 & 70.77 \\
\hline Type of item & D & E & E & F & F & G & G \\
Type de context & MC & MC & OC & WC & MC & WC & MC \\
\hline Item & 8 & 9 & 10 & 11 & 12 & 13 & 14 \\
\hline Average & 78.46 & 87.38 & 88.62 & 82.46 & 84.00 & 74.77 & 80.31 \\
\hline
\end{tabular}

We noticed two items with the highest score averages (over 90). Item 1 corresponds to Type A, which means a Motion Context description to be interpreted in the velocity graph. Item 4 corresponds to type $\mathrm{B}$, which means that given a description of a magnitude in Other Context (no motion) it should be interpreted in the magnitude graph.

It is also noticeable two items with the lowest scores (under 55). The items 5 and 6 correspond to Type $\mathrm{C}$, which means that both present the posing information through the graph of the rate of change of a magnitude, in motion context or in other real context. The options are presented in a text description; to answer correctly, they request information from that graph to interpret the magnitude behavior.

Being each item associated with a Type classification (A-G), as well as context (MC, OC, WC), the scores allow drawing conclusions from a different angle. In Table 6 we observe the average scores grouped together by item Type. There are seven types having an average greater than 70 points, except Type C.

Table 6.

Scores for item type.

\begin{tabular}{cccccc}
\hline \multicolumn{5}{c}{ Scores for item type } & \\
\multirow{2}{*}{ Item posing information: } & Context & Derivative & Function & \\
\hline \multirow{2}{*}{$\begin{array}{c}\text { Options } \\
\text { posing } \\
\text { information }\end{array}$} & Context & & $44.77(\mathrm{C})$ & $88.00(\mathrm{E})$ & $77.54(\mathrm{G})$ \\
\cline { 2 - 6 } & Derivative & $78.92(\mathrm{~A})$ & & $83.23(\mathrm{~F})$ & \\
\cline { 2 - 6 } & Function & $78.62(\mathrm{~B})$ & $74.62(\mathrm{D})$ & \\
\end{tabular}

This redistribution of scores, highlights as a difficult task for students to interpret information from the graph of the rate of change of a magnitude. As opposed, the simplest task is pointed out with item type $\mathrm{E}$, which request to interpret information from the graphical representation of a magnitude. These make us reflect that students can get to interpret correctly the information of the magnitude' behavior from the graph of the magnitude, but to interpret this from the rate of change graph, is not an easy task. In order to describe the behavior of the magnitude from the graph of its rate of change, it is more than just the interpretation of graphs what is needed. Students could interpret graphs, but interpret the graph of a rate of change is not enough to transform this information in the behavior of the magnitude.

Finally we present figure 6 where we consider the averages for each context and considering the participants as a whole. This image helps to reflect whether the type of context in which a 
particular item is presented influences the way in which the students answer. This is relevant because the mathematical knowledge has been introduced to students through the context of linear motion, while the assessment instrument presents magnitudes that are changing in a variety of real contexts. Although there are different contexts for distinct items, the type of information needed to answer them is essentially equivalent.

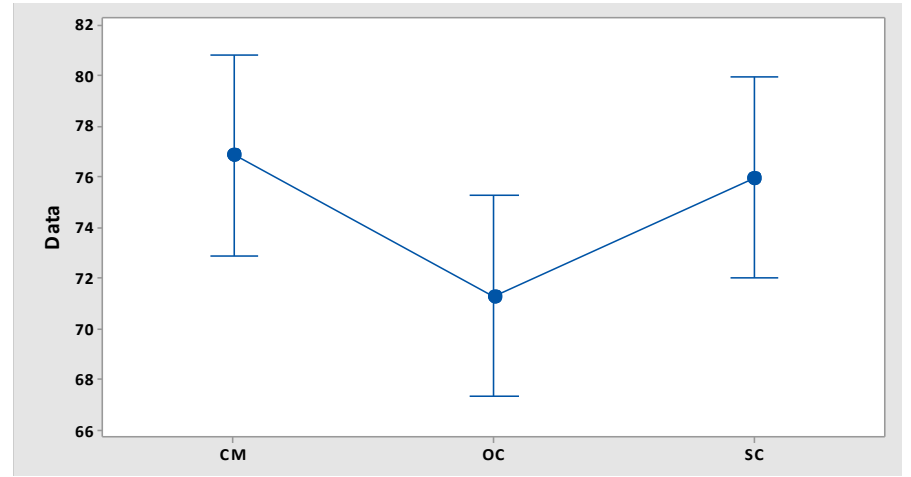

Fig. 6. Averages for context type considering groups together.

As shown in the image, the Motion Context type average (76.88) is slightly greater than the average for Without Context type (76.00); whereas it is clearly greater than the average of the Other Context type (71.31). The comparative analysis done shows that the average for type Other Context results significantly lower than the averages obtained for the other two categories. Moreover, analysis of the difference between those two types resulted not significant.

The latter information is important for us in three ways. First, since the Motion Context became a familiar scenario for students through SimCalc, it is understandable that average in this category resulted greater than in the others. Second, the results of the Motion Context and Without Context being equivalent, make us reflect that the relations the students learned in the context of motion, were applied when answering items where no real context was present, but just the mathematical one. The motion context offered by SimCalc seems to provide graphical relations between the velocity graph and position graph that could be identified in items without a real context, despite can be argued that the latter require a higher level of abstraction. Finally, the difference between the averages of the Motion and Other Context make us reflect that real contexts (different from the motion context) describing situations in which a magnitude is changing, influence in a particular way to recognize and apply the relations between function and derivative addressed in the motion context. Even when the didactic sequence could allow students to apply the relations between function and derivative without context, the transference to other contexts has to consider another elements.

\section{Discussion}

Through this study, we considered the impact of the students' interaction with SimCalc software when it has been integrated to a didactic sequence in which the appropriation of graphic relations between a function and its derivative is promoted. The design of an assessment instrument was discussed, where items were classified by two categories, allowing the interpretation of results from different perspectives. 
When analyzing the responses to items depending on the type of context in which they are presented (motion, other real contexts, and without real context), the results suggest that the graphical relations that students appropriated through the motion context, are not necessarily recognized in other real contexts. Possibly it is required to deepen with the students in certain aspects of these magnitudes in order to identify those relations between a magnitude and its rate of change.

Moreover, the findings suggest that relations appropriated in the motion context can be identified and applied in a purely mathematical context. This finding is didactically relevant in the sense that motion context supports the appropriation of relations between function and derivative having a meaning associated with those mathematical results. The context of linear motion is a suitable scenario for addressing the graphical relations, then the transference to mathematical results could be easily done, and finally, those results should be then discussed in other real contexts trying to foster their application.

This makes us reflect in the importance for mathematical knowledge in Calculus to be achieved first through the suitable scenario of linear motion. Instead of considering it as one of the applications of Calculus theory, our concern is to make Calculus theory an outcome of the analysis of the motion scenario. This scenario has proven to be a suitable medium to bring students a learning environment with meaning, and this environment, without a doubt, is being improved nowadays with the interaction through digital technology as SimCalc.

\section{References}

1. Hillman, T. (2011). The inscription, translation and re-inscription of technology for mathematical learning. Technology, Knowledge and Learning, 16(2), 103-124. doi:10.1007/s10758-011-9182-1

2. Alanís, J. A., \& Salinas, P. (2010). Cálculo de una variable: Acercamientos newtoniano y leibniziano integrados didácticamente. El Cálculo Y Su Enseñanza, 2, 1-14.

3. Salinas, P., Alanís, J. A., \& Pulido, R. (2011). Cálculo de una variable: Reconstrucción para el aprendizaje y la enseñanza. Didac, (56-57), 62-69.

4. Salinas, P., \& Alanís, J. A. (2009). Hacia un nuevo paradigma en la enseñanza del Cálculo dentro de una institución educativa. Revista Latinoamericana de Investigación En Matemática Educativa, 12(3), 355382.

5. Noss, R., Hoyles, C., Mavrikis, M., Geraniou, E., Gutierrez-Santos, S., \& Pearce, D. (2009). Broadening the sense of "dynamic": A microworld to support students' mathematical generalisation. ZDM-The International Journal on Mathematics Education, 41(4), 493-503. doi:10.1007/s11858-009-0182-8

6. Salinas, P., Quintero, E., \& González-Mendívil, E. (2014). An environment to promote a visual learning of Calculus. In H. R. Arabnia, A. Bahrami, L. Deligiannidis, \& G. Jandieri (Eds.), Proceedings of the International Conference on Frontiers in Education: Computer Science and Computer Engineering (pp. 425-429). Las Vegas, Nevada: CSREA Press.

7. Burke, J., Hegedus, S., \& Robidoux, R. (2013). Reflections on significant developments in designing SimCalc software. In S. Hegedus \& J. Roschelle (Eds.), The SimCalc vision and contributions (pp. 65-83). Dordrecht: Springer Netherlands. doi:10.10071978-94-007-5696-0_5

8. Vygotsky, L. (1978). Mind in society: The development of higher psychological processes. (M. Cole, V. John-Steiner, S. Scribner, \& E. Souberman, Eds.). Cambridge, Massachusetts: Harvard University Press.

9. Wertsch, J. V. (1998). Mind as action. New York: Oxford University Press.

10. Rogoff, B. (1993). Aprendices del pensamiento: El desarrollo cognitivo en el contexto social. Barcelona, España: Paidós. 
11. Hegedus, S., \& Moreno-Armella, L. (2010). Accommodating the instrumental genesis framework within dynamic technological environments. For the Learning of Mathematics, 30(1), 26-31.

12. Moreno-Armella, L., \& Hegedus, S. (2009). Co-action with digital technologies. ZDM-The International Journal on Mathematics Education, 41(4), 505-519. doi:10.1007/s11858-009-0200-x

13. Salinas, P., \& Quintero, E. (2013). Integrating digital technology for the innovation of Calculus curriculum. In ASEE Annual Conference and Exposition (pp. 1-14). Atlanta, GA: American Society for Engineering Education.

14. Salinas, P., Alanís, J. A., Pulido, R., Santos, F., Escobedo, J. C., \& Garza, J. L. (2012). Cálculo aplicado: Competencias matemáticas a través de contextos (p. 435). D.F., México: Cengage Learning.

15. Vogt, P. W. (2007). Quantitative research methods for professionals. Boston, MA: Pearson / Allyn and Bacon. 